\title{
Sparse representation of two- and three-dimensional images with fractional Fourier, Hartley, linear canonical, and Haar wavelet transforms
}

\author{
Aykut Koç a,*, Burak Bartan ${ }^{\mathrm{a}, \mathrm{b}}$, Erhan Gundogdu ${ }^{\mathrm{a}}$, Tolga Çukur ${ }^{\mathrm{c}, \mathrm{d}}$, Haldun M. Ozaktas ${ }^{\mathrm{c}}$ \\ a Intelligent Data Analytics Research Program Dept. Aselsan Research Center, Ankara, Turkey \\ ${ }^{\mathrm{b}}$ Department of Electrical Engineering, Stanford University, CA, USA \\ ${ }^{\mathrm{c}}$ Department of Electrical and Electronics Engineering, Bilkent University, Ankara, Turkey \\ ${ }^{\mathrm{d}}$ National Magnetic Resonance Research Center (UMRAM), Bilkent University, Ankara, Turkey
}

\section{A R T I C L E I N F O}

\section{Article history:}

Received 1 November 2016

Revised 3 January 2017

Accepted 25 January 2017

Available online 3 February 2017

\section{Keywords:}

Compressibility

Image representation

Sparsifying transforms

Transform domain coding

Haar wavelet transform

Fractional Fourier transform

Simplified fractional Hartley transform

Linear canonical transforms

\begin{abstract}
A B S T R A C T
Sparse recovery aims to reconstruct signals that are sparse in a linear transform domain from a heavily underdetermined set of measurements. The success of sparse recovery relies critically on the knowledge of transform domains that give compressible representations of the signal of interest. Here we consider two- and three-dimensional images, and investigate various multi-dimensional transforms in terms of the compressibility of the resultant coefficients. Specifically, we compare the fractional Fourier (FRT) and linear canonical transforms (LCT), which are generalized versions of the Fourier transform (FT), as well as Hartley and simplified fractional Hartley transforms, which differ from corresponding Fourier transforms in that they produce real outputs for real inputs. We also examine a cascade approach to improve transform-domain sparsity, where the Haar wavelet transform is applied following an initial Hartley transform. To compare the various methods, images are recovered from a subset of coefficients in the respective transform domains. The number of coefficients that are retained in the subset are varied systematically to examine the level of signal sparsity in each transform domain. Recovery performance is assessed via the structural similarity index (SSIM) and mean squared error (MSE) in reference to original images. Our analyses show that FRT and LCT transform yield the most sparse representations among the tested transforms as dictated by the improved quality of the recovered images. Furthermore, the cascade approach improves transform-domain sparsity among techniques applied on small image patches.
\end{abstract}

(c) 2017 Elsevier Ltd. All rights reserved.

\section{Introduction}

Classical sampling theory dictates that signals sampled at twice the rate of their effective bandwidth can be recovered perfectly through linear algorithms. This Nyquist-sampling criterion requires acquisition of an often impractically large number of measurements. In contrast, the recent theory of sparse recovery suggests that signals can be recovered from a much smaller set of measurements under the condition that they have sparse representations (Donoho, Elad, \& Temlyakov, 2006). The promise of significantly fewer measurements has led sparse recovery to receive ample interest in multiple domains including image processing and medical imaging (Lustig, Donoho, \& Pauly, 2007). Of course, a critical step

\footnotetext{
* Corresponding author.

E-mail addresses: aykutkoc@aselsan.com.tr (A. Koç), bbartan@stanford.edu (B. Bartan), egundogdu@aselsan.com.tr (E. Gundogdu), cukur@ee.bilkent.edu.tr (T. Çukur), haldun@ee.bilkent.edu.tr (H.M. Ozaktas).
}

to successful recovery is the specification of linear transform domains in which the signals are sparse or highly compressible, i.e., represented with few non-zero coefficients (Candès \& Romberg, 2007). Yet, maximally-sparse transforms remain largely unexplored in many application domains, compromising recovery performance.

Efficient representation of digital images is a prevalent field that has implications for both storage and transmission of visual information. Naturally, certain aspects of image representation such as compression, quantization and coding have been broadly studied in literature (Leung and Taubman, 2005; Pennebaker and Mitchell, 1992; Song, Peng, Xu, Shi, and Wu, 2015; ISO/IEC, 2002; Suzuki \& Ikehara, 2010; Zhu \& Chen, 2012). These efforts put forth Wavelet and Fourier-related transforms such as discrete cosine transform (DCT) as an established standard in image compression (Pennebaker and Mitchell, 1992; ISO/IEC, 1992; 2002). Note that the problem of sparse recovery differs conceptually from image compression. In compression, an encoder first reduces the dimensionality of fully-sampled images, and based on the encoding 


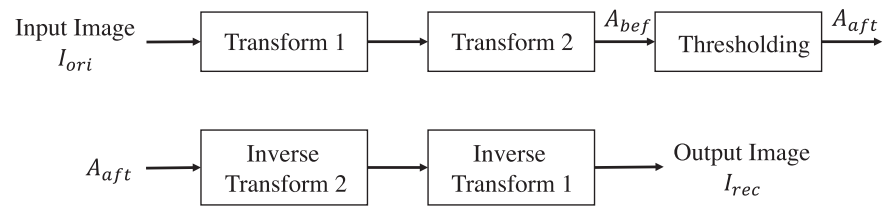

Fig. 1. The following stages are performed sequentially during the sparse recovery experiments: sparsifying transformations, thresholding of varying percentages of transform-domain coefficients, inverse tranformation to reconstruct images.

algorithm a decoder then restores the original image. In sparse recovery, however, the algorithm (or equivalently the transform) that yields optimally sparse representation of the images is unknown. Few recent studies have considered image sparsification based on discrete Hartley transform (DHT) and their fractional versions such as fractional Fourier transform (FRT) and fractional Hartley transform (FRHT) (Divya \& Prabha, 2015; Jimenez, Torres, \& Mattos, 2011; Kumar, Singh, \& Khanna, 2012). However, a comparative evaluation of candidate transforms in comparison to standard approaches is lacking.

Magnetic resonance imaging (MRI) is another area where sparse recovery approaches have been gaining broad attention (Cukur, Lustig, \& Nishimura, 2009; Lustig et al., 2007). Accelerated MRI acquisitions can be performed by undersampling the measurement matrix in the Fourier domain. Compressibility in a linear transform domain is then leveraged to recover unacquired data and reconstruct images. In many studies, MRI images are typically assumed to be compressible directly in the image domain or in the Wavelet domain (Cukur, Lustig, Saritas, \& Nishimura, 2011). However, given the substantial variability across different anatomies in the body, the respective images and the optimal sparsifying transforms remain unknown. There is a pressing need to explore transforms that can be adapted to the intrinsic properties of MR images to enhance sparsity and thereby improve image quality.

Here, we consider the problem of obtaining sparse representation of multi-dimensional images via appropriate sparsifying transforms. To encounter the high degree of variability across image structure in various applications, we examine transforms with one or more free parameters that enable adaptation to specific images. For this purpose, we not only test fractional Fourier and fractional Hartley transforms but also include another integral transform known as linear canonical transform (LCTs). Onedimensional (1D) LCTs (Healy, Kutay, Ozaktas, \& Sheridan, 2016; Ozaktas, Zalevsky, \& Kutay, 2001) constitute a three-parameter class of linear integral transforms that include among its special cases, the one-parameter subclasses of fractional Fourier transforms (FRTs), scaling operations, and chirp multiplication (CM) and chirp convolution (CC, also known as Fresnel transform) operations. For cases with limits on memory or computation power, we also consider a framework based on cascaded transforms applied on small image patches, specifically Hartley-Haar transforms. Discrete Cosine Transform (DCT) and Wavelets with 9/7 and 5/3 filters are also used as references in comparisons. The sparse recovery performance of these different approaches are compared in both two- and three-dimensional images based on mean squared error (MSE) and structural similarity index (SSIM) metrics.

FRT and LCT transforms yield the most sparse representations most of the time among the tested transforms as dictated by the improved quality of the recovered images. Their sparsifying effects as a preprocessing step have also been demonstrated.

The paper is organized as follows: in Section 2, we will give preliminary information about the transforms we used, the general method that we followed in sparsely representing images and present the example images that we used. In Section 3, we will demonstrate our results and finally in Section 4, we will give our discussions and concluding remarks.

\section{Methods}

\subsection{Sparsifying transforms}

\subsubsection{The fractional Fourier transform}

FRT is a generalized version of Fourier transform. The ath order fractional Fourier transform $\left\{\mathcal{F}^{a} f\right\}(u)$ of the function $f(u)$ may be defined for $0<|a|<2$ as

$$
\begin{aligned}
& \mathcal{F}^{a}[f(u)] \equiv\left\{\mathcal{F}^{a} f\right\}(u) \equiv \int_{-\infty}^{\infty} K_{a}\left(u, u^{\prime}\right) f\left(u^{\prime}\right) d u^{\prime} \\
& K_{a}\left(u, u^{\prime}\right) \equiv A_{\phi} \exp \left[i \pi\left(u^{2} \cot \phi-2 u u^{\prime} \csc \phi+u^{\prime 2} \cot \phi\right)\right] \\
& A_{\phi} \equiv \frac{\exp (-i \pi \operatorname{sgn}(\sin \phi) / 4+i \phi / 2)}{|\sin \phi|^{1 / 2}}
\end{aligned}
$$

where $\phi \equiv a \pi / 2$ and $i$ is the imaginary unit. The kernel approaches $K_{0}\left(u, u^{\prime}\right) \equiv \delta\left(u-u^{\prime}\right)$ and $K_{ \pm 2}\left(u, u^{\prime}\right) \equiv \delta\left(u+u^{\prime}\right)$ for $a=0$ and $a= \pm 2$ respectively. The FRT, like the Fourier transform, produces complex outputs for real inputs.
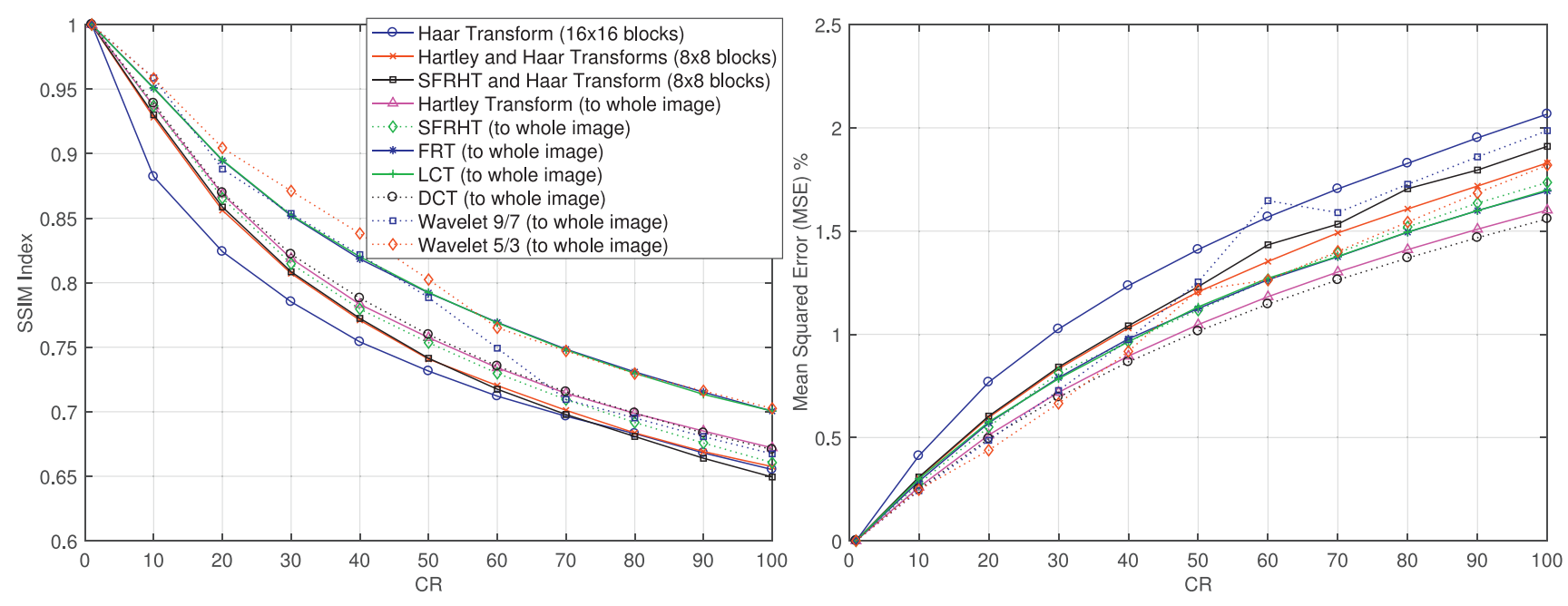

Fig. 2. SSIM index and MSE vs CR for lake image. 

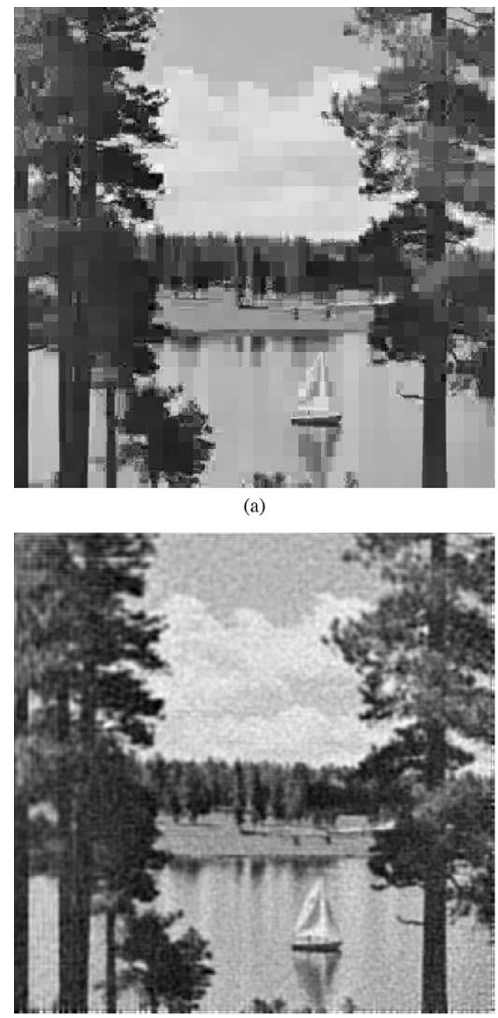

(c)

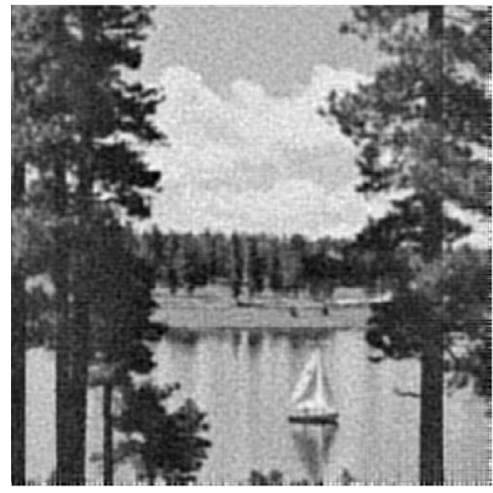

(e)

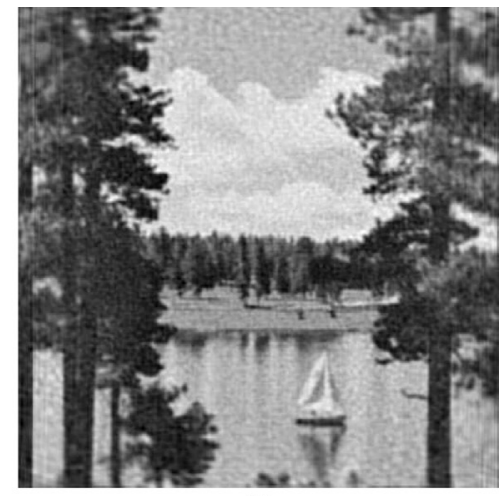

(b)

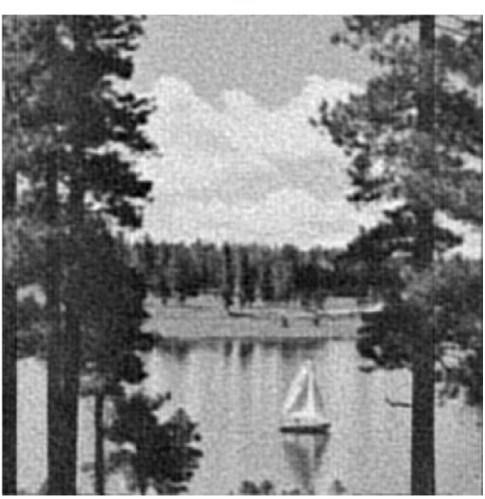

(d)

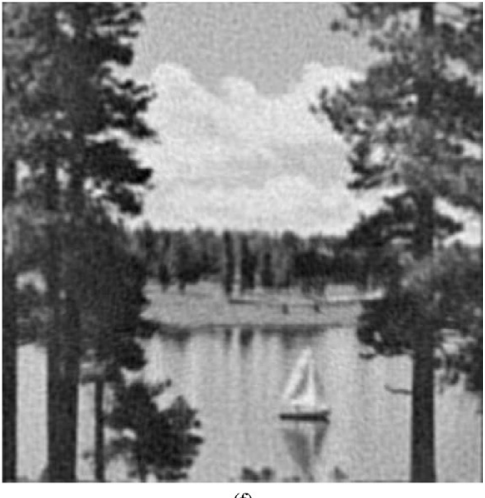

(f)

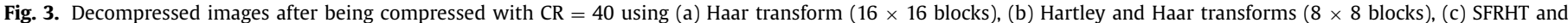

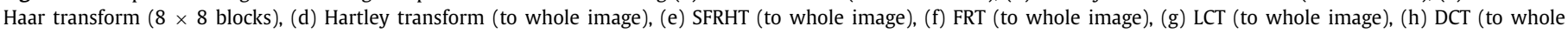
image), (i) Wavelet 9/7 (to whole image), (j) Wavelet 5/3 (to whole image).

\subsubsection{The Hartley transform}

The Hartley transform, however, gives real outputs for real inputs and is related to the Fourier transform as

$H\{x(t)\}=\operatorname{Re}\{F\{x(t)\}\}-\operatorname{Im}\{F\{x(t)\}\}$,

where $H$ and $F$ represent Hartley and Fourier transforms, respectively. The original signal can be recovered by taking the Hartley transform of $H\{x(t)\}$, by recalling that the Hartley transform is an involution.

This basic relationship between Hartley and Fourier transforms does not exist between fractional versions of these transforms. The reason for this is that the FRT does not produce conjugate symmetric outputs for real inputs, unlike the Fourier transform. There is more than one definition for the fractional Hartley transform (FRHT), most of which fail to produce real outputs for real inputs. Simplified fractional Hartley transform, (SFRHT) defined in Pei and Ding (2002), has the property of producing real outputs for real inputs:

$H^{\alpha}(s)=\sqrt{\frac{1}{2 \pi}} \int_{-\infty}^{\infty} \operatorname{cas}(s t) \cos \left(\frac{1}{2} \cot (\phi) t^{2}\right) x(t) d t$,

where $\operatorname{cas}(x)=\cos (x)+\sin (x), \phi=\alpha \pi / 2$ and $\alpha$ is the fractional order of SFRHT.

\subsubsection{The linear canonical transform}

The 1D LCT of $f(u)$ with parameter matrix $\mathbf{M}$ is denoted as $f_{\mathbf{M}}(u)=\left(\mathcal{C}_{\mathbf{M}} f\right)(u)$ :

$$
\begin{aligned}
\left(\mathcal{C}_{\mathbf{M}} f\right)(u)= & \sqrt{\beta} e^{-i \pi / 4} \\
& \times \int_{-\infty}^{\infty} \exp \left[i \pi\left(\alpha u^{2}-2 \beta u u^{\prime}+\gamma u^{\prime 2}\right)\right] f\left(u^{\prime}\right) d u^{\prime},
\end{aligned}
$$

where $\alpha, \beta, \gamma$ are real parameters independent of $u$ and $u^{\prime}$ and where $\mathcal{C}_{\mathbf{M}}$ is the LCT operator. The transform is unitary. For certain values of $\alpha, \beta$, and $\gamma$, LCT reduces to the FRT. 


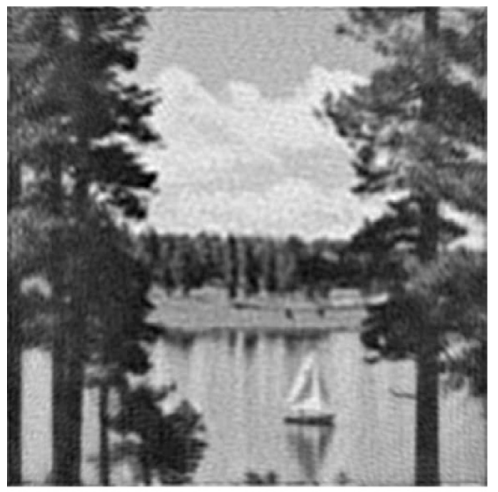

(g)

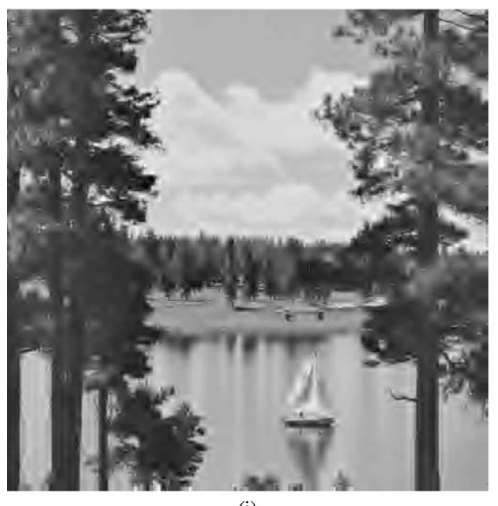

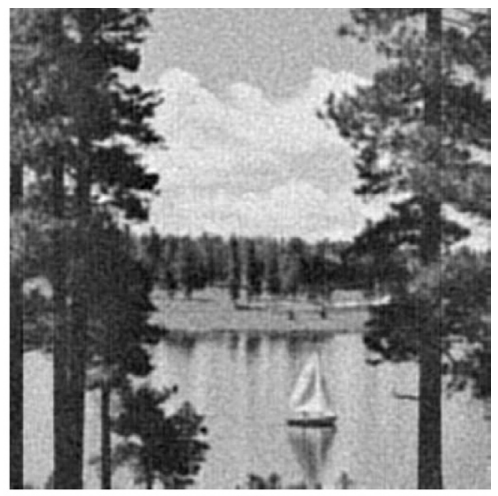

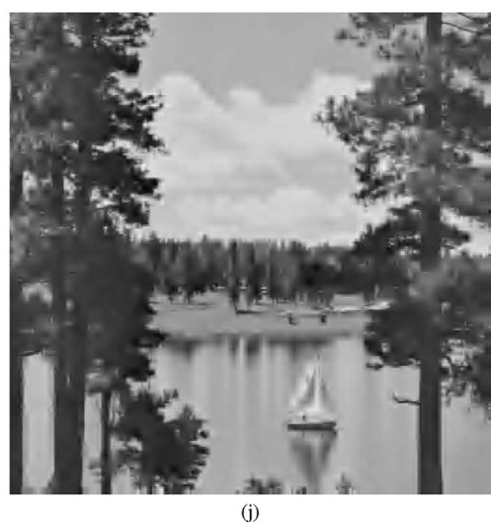

Fig. 3. Continued

Due to the increased degree of freedom they provide, LCTs appear widely in optics (Ozaktas et al., 2001), electromagnetics, classical and quantum mechanics (Healy et al., 2016), in computational and applied mathematics (Koç, Oktem, Ozaktas, \& Kutay, 2016), radar signal processing (Chen, Guan, Huang, Liu, \& He, 2015), speech processing (Qiu, Li, \& Li, 2013) as well image encryption and watermarking (Li \& Shi, 2014; Singh \& Sinha, 2010). The application areas of LCTs also include the study of scattering from periodic potentials, laser cavities, and multilayered structures in optics and electromagnetics. They can also be used for fast and efficient realization of filtering in linear canonical transform domains (Barshan, Kutay, \& Ozaktas, 1997).

Generalizations to two-dimensional (2D) transforms and complex-parametered transforms are also present in the literature. Classification of first-order optical systems and their representation through LCTs are studied in for 1D and 2D cases (Bastiaans \& Alieva, 2007; Healy et al., 2016). Bilateral Laplace transforms, Bargmann transforms, Gauss-Weierstrass transforms, fractional Laplace transforms, and complex-ordered FRTs are all special cases of complex linear canonical transforms (CLCTs) (Healy et al., 2016; Wolf, 1974).

There are fast algorithms that have been proposed for efficient computation of FRT, Hartley transform and LCTs (Hennelly \& Sheridan, 2005; Koc, Ozaktas, \& Hesselink, 2011; Koç, Ozaktas, Candan, \& Kutay, 2008; Koç, Ozaktas, \& Hesselink, 2010a; 2010b; Ozaktas, Arıkan, Kutay, \& Bozdağı, 1996; Ozaktas, Koç, Sari, \& Kutay, 2006), of which existence is critical for the specific applications given in this paper.

\subsubsection{Cascade transforms}

Here we examine a cascade-transform method where Transform 1 can be selected as either the Hartley transform or the SFRHT and Transform 2 is strictly constrained to be the Haar wavelet transform. Note that Haar wavelet transforms are not computed for the whole image at once but in each $(\mathrm{NxN})$ block separately, where $\mathrm{N}$ is usually 8 or 16 . Furthermore, because the Haar wavelet transform can only implement real-to-real mapping, it constrains Transform 1 to also be selected as a real-to-real transform. As a result, FRT and LCT transforms are not considered in this cascade form.

\subsection{Sparse recovery}

The sparse recovery experiment conducted here is summarized in Fig. 1. In this experiment, original images are first transformed via one of the candidate methods described in previous sections. In the second stage, the subset of the transform-domain coefficients that are smaller then a select threshold is set to zero. Finally, an inverse transform is performed on the thresholded coefficients to recover the image. The threshold selection reflects a desired rate of undersampling denoted here as CR:

$C R=\frac{\left\|V_{b e f}\right\|_{0}}{\left\|V_{a f t}\right\|_{0}}$

where $\|x\|_{0}$ denotes the $l_{0}$-norm of vector $x$, defined as the number of nonzero elements in $x$. Note that $V_{b e f}=\left[A_{b e f, 1}^{T} A_{b e f, 2}^{T} \ldots A_{b e f, N}^{T}\right]^{T}$ and $V_{a f t}=\left[A_{a f t, 1}^{T} A_{a f t, 2}^{T} \ldots A_{a f t, N}^{T}\right]^{T}$, where $A_{b e f, i}$ and $A_{a f t, i}$ are the $i$ th columns of $A_{b e f}$ and $A_{a f t}$, which correspond to the transform of the ideal reference image $I_{o r i} \in \mathrm{IR}^{M \times N}$ before and after the thresholding, respectively. So, $A=\mathcal{T}\left\{I_{\text {ori }}\right\}$, where $\mathcal{T}$ is the transform that we are using. CR corresponds to the ratio of nonzero elements in the transformed data before and after the thresholding is applied.

Comparative assessments of sparsifying transforms were based on two image quality metrics: mean squared error (MSE) and structural similarity index (SSIM). For each method, MSE was 

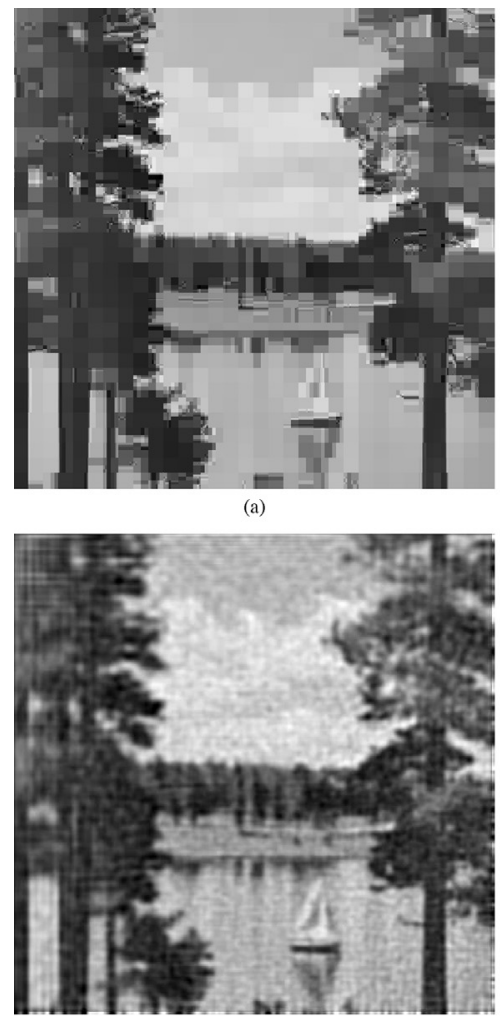

(c)

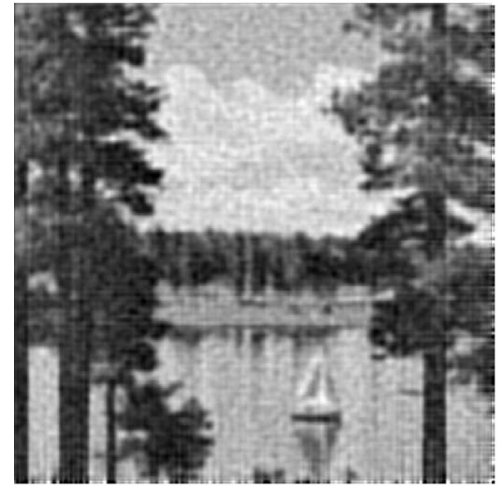

(e)

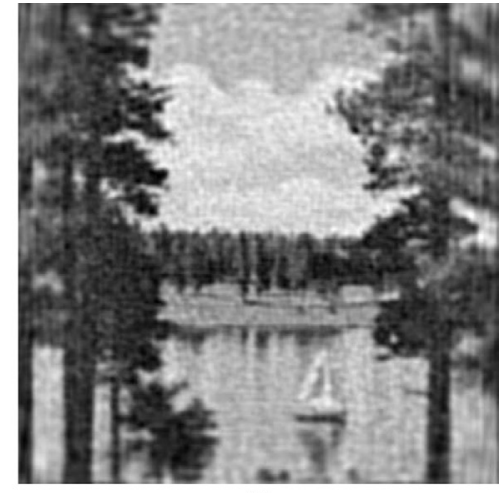

(b)

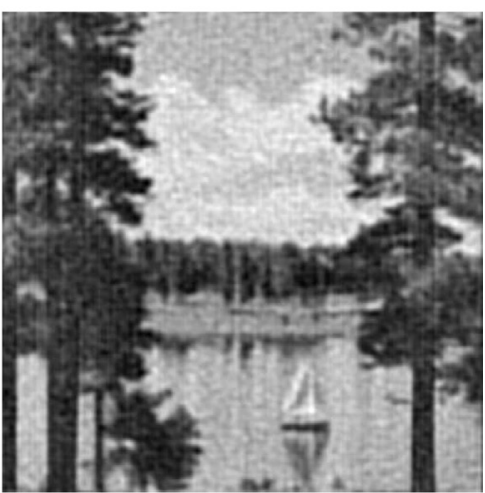

(d)

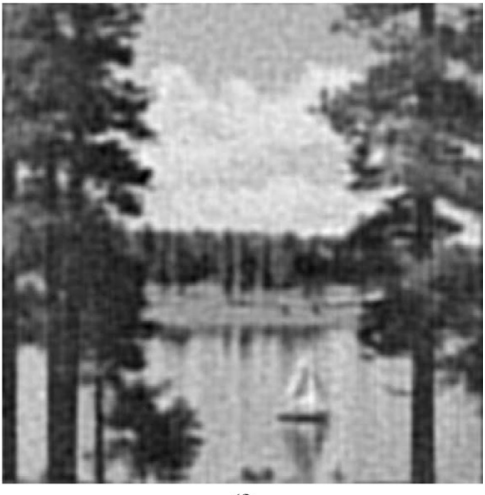

(f)

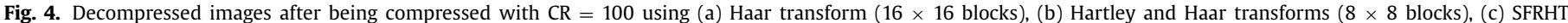

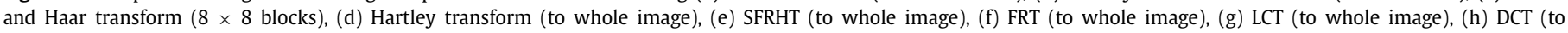
whole image), (i) Wavelet 9/7 (to whole image), (j) Wavelet 5/3 (to whole image).

calculated as follows:

$M S E=\frac{\sum_{j=1}^{M} \sum_{i=1}^{N}\left(I_{r e c}(i, j)-I_{\text {ori }}(i, j)\right)^{2}}{\sum_{j=1}^{M} \sum_{i=1}^{N}\left(I_{\text {ori }}(i, j)\right)^{2}} \times 100$,

where $I_{\text {ori }} \in \mathrm{IR}^{M \times N}$ is the ideal reference image, $I_{\text {rec }} \in \mathrm{IR}^{M \times N}$ is the recovered image, and $I_{o r i}(i, j), I_{\text {rec }}(i, j)$ are the image pixels. An average SSIM metric was calculated across the whole image as follows (Wang, Bovik, Sheikh, \& Simoncelli, 2004):

$S S I M=\frac{1}{X Y} \sum_{x=1}^{X} \sum_{y=1}^{Y} \frac{\left(2 \mu_{x} \mu_{y}+c_{1}\right)\left(2 \sigma_{x y}+c_{2}\right)}{\left(\mu_{x}^{2}+\mu_{y}^{2}+c_{1}\right)\left(\sigma_{x}^{2}+\sigma_{y}^{2}+c_{2}\right)}$

where $x$ and $y$ are image patches extracted from the recovered and ideal images, respectively. $\mu$ and $\sigma$ are the mean and covariance of pixel intensity within the given patches. The remaining parameters were selected as $c_{1}=10^{-4}, c_{2}=9 \times 10^{-4}$, a Guassian kernel of width 10 , standard deviation 5 .
All methods were implemented in MATLAB (Mathworks Inc) and performed on a PC with $2.7 \mathrm{GHz}$ Intel i5 CPU and 8 GB RAM. For fractional transforms and the linear canonical transform, transform parameters were optimized to optimize image quality after sparse recovery. This optimization enabled unbiased comparisons among techniques.

\subsection{Analyzed images}

The proposed methods have been applied to representative two-dimensional (2D) and three-dimensional (3D) images. The sample 2D image depicted an outdoors visual scene comprising a lake and surrounding trees (from an online database at http: //www.imageprocessingplace.com/root_files_V3/image_databases. $\mathrm{htm})$. This grayscale image size was of size $512 \times 512$. The sample 3D image was taken as an MRI dataset acquired in the lower extremities. Lower leg angiograms were collected on a $1.5 \mathrm{~T}$ scanner 


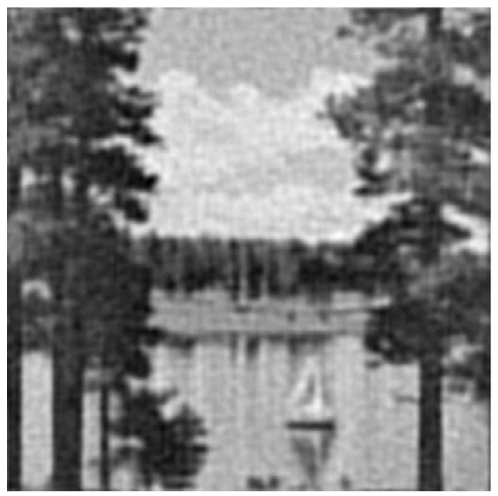

(g)

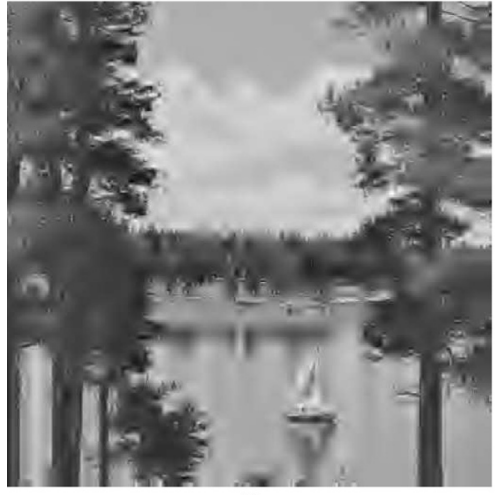

(i)

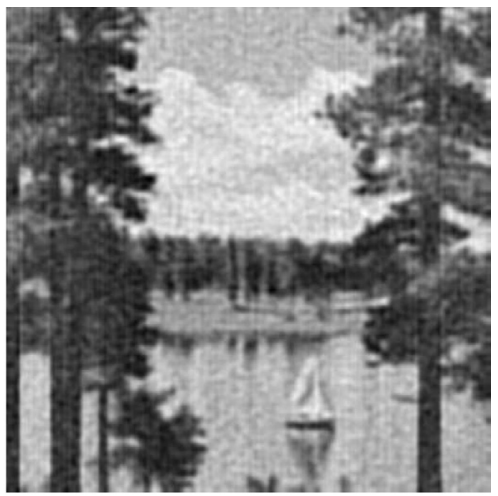

(h)

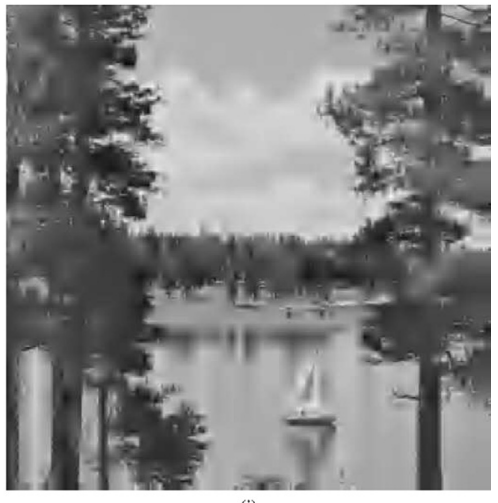

(j)

Fig. 4. Continued

(a)

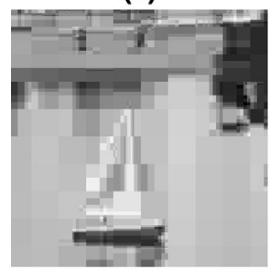

(f)

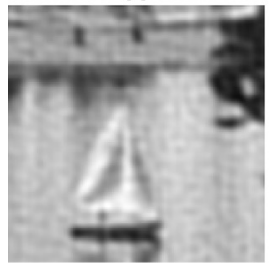

(b)

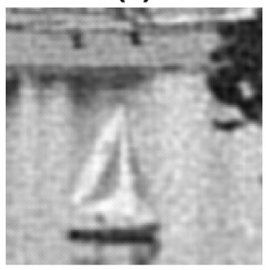

(g)

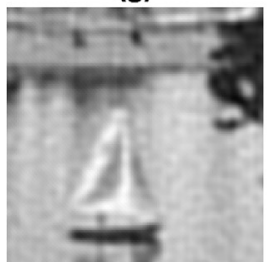

(c)

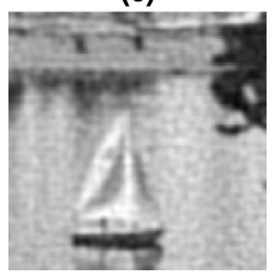

(h)

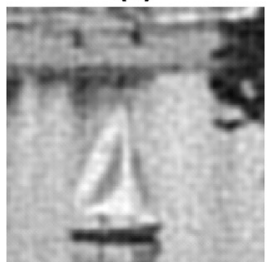

(d)

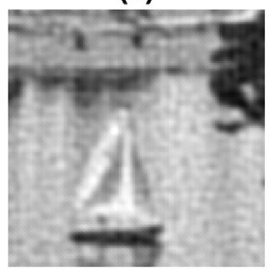

(i)

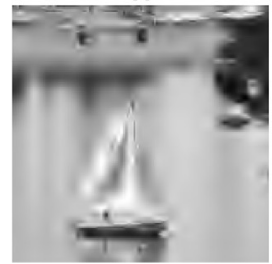

(e)

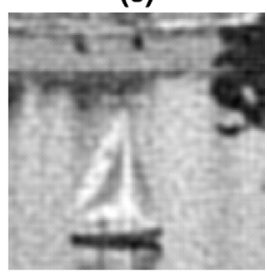

(j)

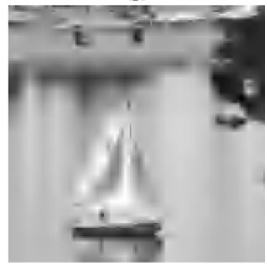

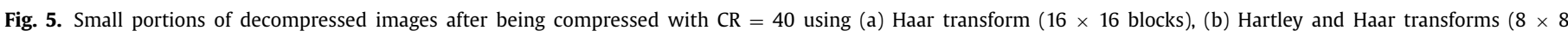

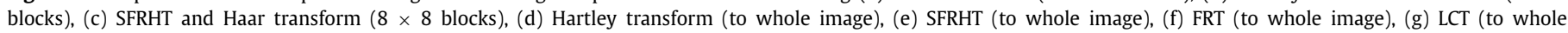
image), (h) DCT (to whole image), (i) Wavelet 9/7 (to whole image), (j) Wavelet 5/3 (to whole image).

via a magnetization-prepared steady-state sequence (Cukur, Lustig et al., 2011; Cukur, Shimakawa et al., 2011b). The following protocol was prescribed: a field-of-view of $192 \times 128 \times 128 \mathrm{~mm}^{3}$, an isotropic spatial resolution of $1 \mathrm{~mm}$, a repetition time of $4.6 \mathrm{~ms}$, a scan time of $52 \mathrm{~s}$, and 4 interleaves with 4096 phase encodes each.

\section{Results}

We have performed experiments by using both twodimensional and three-dimensional images.

\subsection{Two-dimensional images}

To observe the behavior of the proposed method and to compare it with other methods, a wide range of values for compression ratio (between 1 and 100) is spanned. Fig. 2 demonstrates how the image quality measurement parameters SSIM and MSE change with respect to different compression ratios for our proposed transforms as well as standard Discrete Cosine transform (DCT) and Wavelet based methods with 9/7 and 5/3 filters. The test image for these simulation results is rich in terms of high frequency components. Moreover, the decompressed output images for com- 
(a)

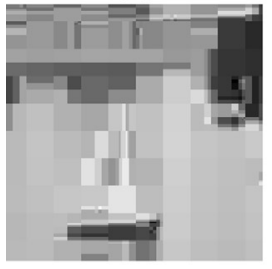

(f)

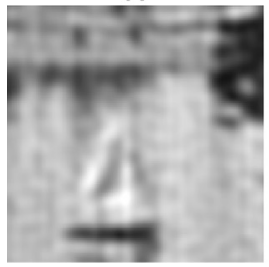

(b)

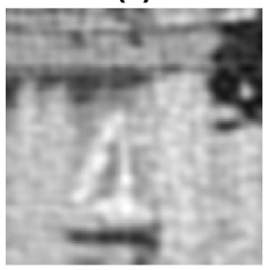

(g)

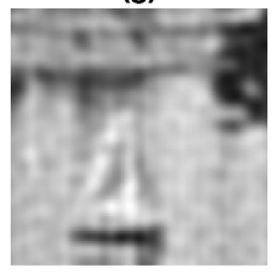

(c)

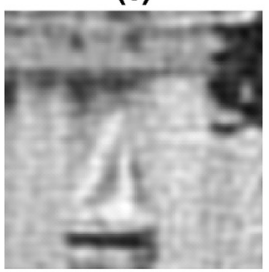

(h)

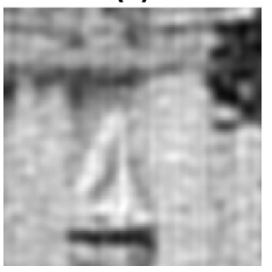

(d)

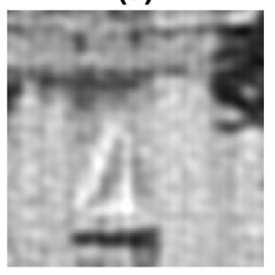

(i)

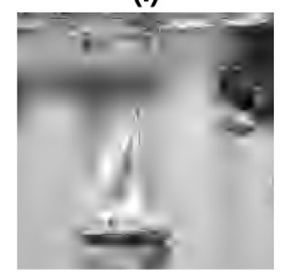

(e)

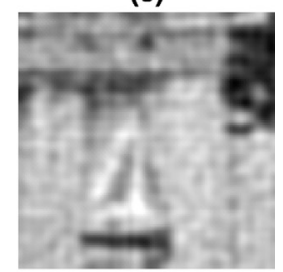

(j)

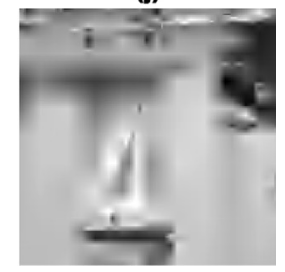

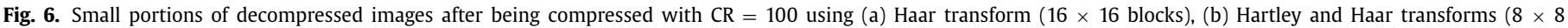

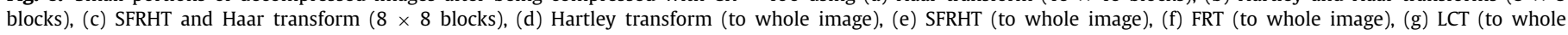
image), (h) DCT (to whole image), (i) Wavelet 9/7 (to whole image), (j) Wavelet 5/3 (to whole image).

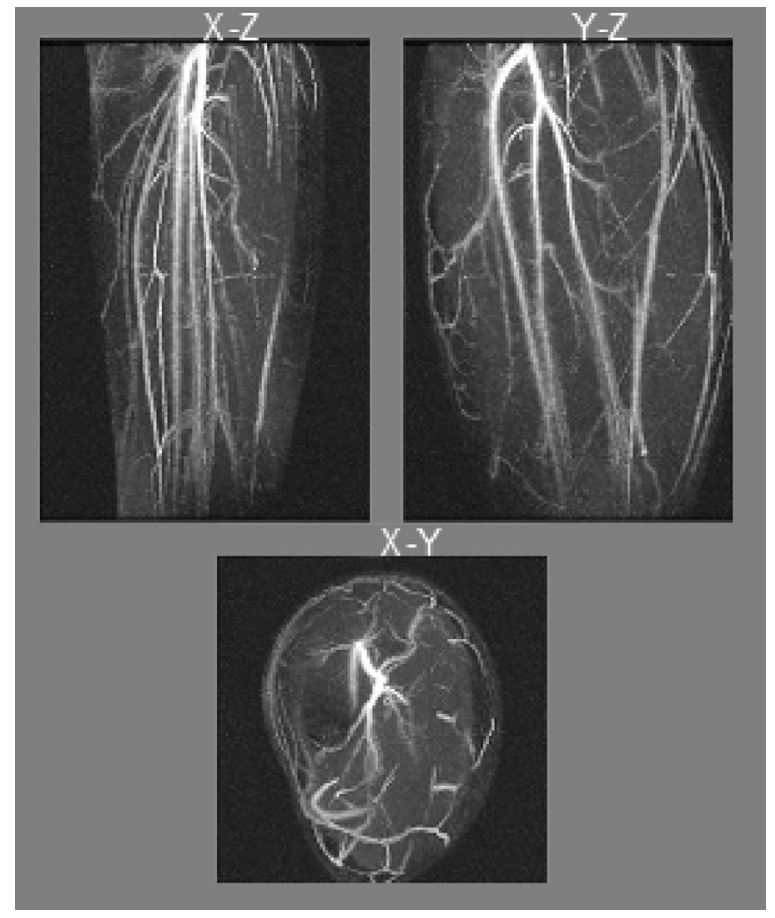

Fig. 7. The three-dimensional angiographic images of the lower leg were processed with maximum-intesntiy projections to visualize the underlying vasculature. Sagittal, coronal and axial projections are shown.

pression ratios of 40 and 100 can also be seen in Figs. 3 and 4, respectively.

Whenever Haar wavelet transform is used, block size is varied, taking the values of 8,16 , or 32 and the best one is shown in the plots in Fig. 2. Also, for fractional transforms and linear canonical transform, the optimal transform parameters are found and the results of the corresponding output are shown in the plots. Optimization of the transform parameters is performed by simply sweeping parameters and finding the parameter that yields the highest SSIM index as we aim to understand the performance of the transforms in how much they can sparsify the data without causing significant distortion in the image. To be more specific, we first sweep the parameter range in 10 equal parts, find the best performing parameter, then further sweep in the neighborhood of this coarse optimal value with 10 more sub-steps and find the optimal transform parameter. Furthermore, for the wavelet transforms with different levels, we find and use the level at which the transform reaches its maximum SSIM index.

Simulation results show that the performance of Haar wavelet transform can be increased if Hartley transform or SFRHT is first applied to the input image. However, applying only Hartley transform is observed to yield better results compared to the combination of Hartley and Haar wavelet transforms. This would mean that it is preferable to use Hartley transform alone in this case.

Wavelet transform with filter 5/3 leads to higher SSIM indices for CR values up to 50 . For CR values larger than 50 , we see that the performances of FRT and LCT catch up with the wavelet transform with filter $5 / 3$. Moreover, these three transforms seem to outperform DCT, in terms of SSIM index, for a wide range of CRs when all of them are applied to the whole image.

A comparison between only FRT and LCT shows that their performances are nearly identical. This implies that it would be more preferable to use FRT instead of LCT since LCT requires optimization of three parameters whereas FRT requires only one.

Figs. 3 and 4 show that the deterioration when only Haar wavelet transform is used is more likely to bother human-eye compared to other methods because blocks of the chosen size $\mathrm{N}$ can be noticed in the output images. Nonetheless, applying Hartley transform before Haar wavelet transform alleviates this problem. To better assess the quality of images and the effects of different transforms on sparsified data visually, a small patch of the recovered test image has been zoomed and presented in Figs. 5 and 6.

DCT and Wavelet based methods are very essential and established in the image compression field. This has led to a great body of research on their fast implementations for years. Consequently, there exist optimized codes for DCT and Wavelets, including low-level implementations that boost efficiency. Keeping this in mind, it would not be fair to make processing time comparisons between DCT/Wavelet based methods and the proposed ones. However, there are $O(N \log N)$ time efficient digital implementation algorithms for both FRT and LCTs (Koç et al., 2008; Ozaktas et al., 1996). Although these algorithms are not exhaustively optimized at low-level, their general algorithmic complexity is on the same order with those of the DCT and Wavelets. Therefore, the proposed 


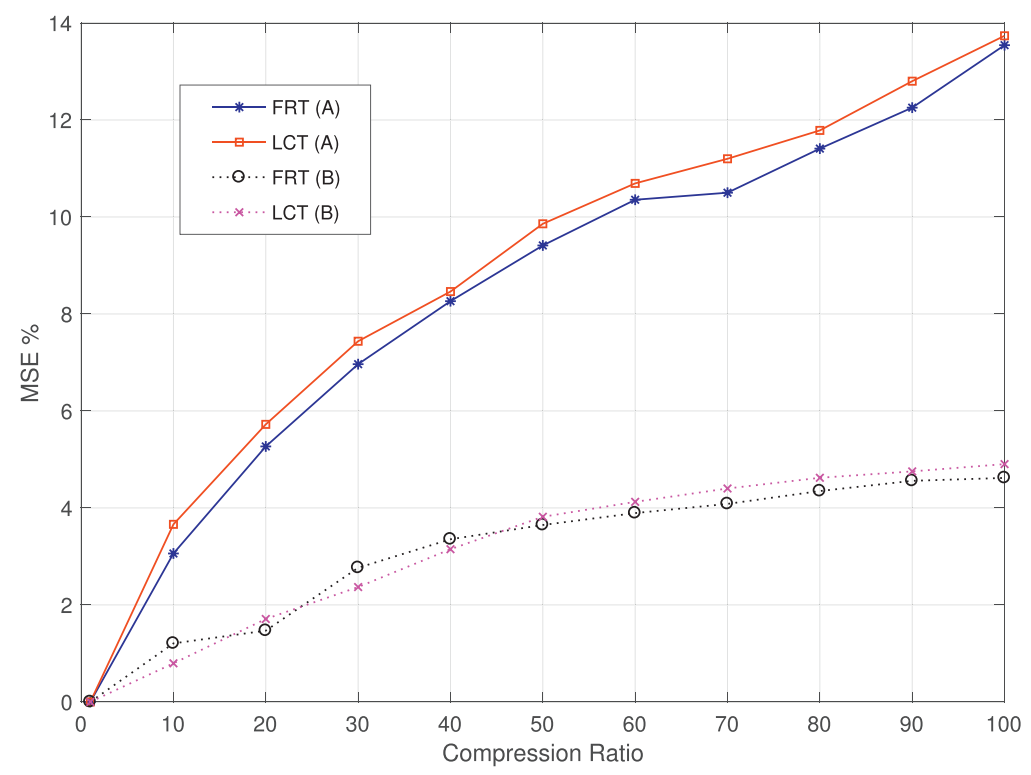

Fig. 8. MSE vs CR for the three-dimensional lower-leg image.

methods in this paper can also be implemented with increased efficiency, depending on the specific application.

\subsection{Three-dimensional images}

Three-dimensional images are frequently used in medical imaging and the angiographic dataset analyzed in this study is shown in Fig. 7. For visualization only, the $192 \times 128 \times 128$ complex-valued image was processed with a maximum-intensity projection in three cardinal dimensions. During the actual analyses, we have experimented only with FRT and LCT since they are capable of handling complex values.

We have compressed the three-dimensional image using two different approaches:

1. Regarding the three-dimensional image as a set of twodimensional images and finding an optimal fractional order for each layer and compressing each layer independently

2. Taking the three-dimensional transform of the image data and treating the resulting data as a three-dimensional signal to compress

The performances of these two different approaches when FRT or LCT is used are shown in Fig. 8. In Fig. 8, FRT (A) and LCT (A) correspond to the cases where the first approach is used. Likewise, FRT (B) and LCT (B) means that these transforms are applied to the image using the second approach.

Fig. 8 illustrates that LCT and FRT perform similarly. However, the second approach produces higher quality outputs for three-dimensional images compared to the first approach.

\section{Conclusion and future work}

In this study, we investigated several different transforms or cascades of transforms with respect to their effects on the sparsity of image data in the respective domains. Our results indicate that, overall, the fractional and linear canonical transforms with tunable parameters offer improved sparsity compared to static Fourier and Wavelet transforms for both 2D and 3D images. This difference in transform-domain sparsity is reflected in sparse recovery performance, and the differences in performance become more prominent at higher compression ratios. The closest competitor to fractional and linear canonical transforms is the Hartley transform applied to the whole image. Note that in practice there may be constraints of computing time or memory, which may motivate the use of transforms on small-image patches. Our analyses show that, in such cases, the cascade Haar-Hartley transform yields improved sparsity and recovery performance compared to other approaches. Additionally, the existence of $O(N \log N)$ time algorithms for digital computation of FRTs and LCTs makes these transforms practical in terms of the processing time considerations. Lastly, we find that 3D transforms yield substantially improved performance on 3D images compared to 2D transforms applied on each cross-section independently. This leads to a single transform-parameter specification for the entire 3D volume. In future work, we plan to investigate a sliding-window approach where stacks of 2D images are processed with 3D transforms, and the transform parameters are adaptively optimized for each stack.

\section{Acknowledgments}

The work of T. Çukur was supported in part by a Marie Curie Actions Career Integration Grant (PCIG13-GA-2013-618101), by a European Molecular Biology Organization Installation Grant (IG 3028), and by a TUBA GEBIP fellowship. H. M. Ozaktas acknowledges partial support of the Turkish Academy of Sciences.

\section{References}

Barshan, B., Kutay, M. A., \& Ozaktas, H. M. (1997). Optimal filtering with linear canonical transformations. Optics Commun., 135(1-3), 32-36.

Bastiaans, M. J., \& Alieva, T. (2007). Classification of lossless first-order optical systems and the linear canonical transformation. Journal of Optical Society of America $A, 24$.

Candès, E., \& Romberg, J. (2007). Sparsity and incoherence in compressive sampling. Inverse Problems, 23(3), 969-985.

Chen, X., Guan, J., Huang, Y., Liu, N., \& He, Y. (2015). Radon-linear canonical ambiguity function-based detection and estimation method for marine target with micromotion. IEEE Transactions on Geoscience and Remote Sensing, 53(4), 2225-2240.

Cukur, T., Lustig, M., \& Nishimura, D. G. (2009). Improving non-contrast-enhanced steady-state free precession angiography with compressed sensing. Magnetic Resonance in Medicine, 61(5), 1122-1131.

Cukur, T., Lustig, M., Saritas, E. U., \& Nishimura, D. G. (2011a). Signal compensation and compressed sensing for magnetization-prepared MR angiography. IEEE Transactions on Medical Imaging, 30(5), 1017-1027. 
Cukur, T., Shimakawa, A., Yu, H., Hargreaves, B. A., Hu, B. S., Nishimura, D. G., \& Brittain, J. H. (2011b). Magnetization-prepared IDEAL bSSFP: A flow-independent technique for noncontrast-enhanced peripheral angiography. Journal of Magnetic Resonance Imaging, 33(4), 931-939.

Divya, B., \& Prabha, K. (2015). 2D-discrete frac. fourier transform for facial image compression. International Journal of Inventions in Computer Science and Engineering, 2, 2348-3431.

Donoho, D. L., Elad, M., \& Temlyakov, V. N. (2006). Stable recovery of sparse overcomplete representations in the presence of noise. IEEE Transactions on Information Theory, 52(1), 6-18.

Healy, J. J., Kutay, M. A., Ozaktas, H. M., \& Sheridan, J. T. (2016). Linear canonical transforms: Theory and applications. New York, NY: Springer New York.

Hennelly, B. M., \& Sheridan, J. T. (2005). Fast numerical algorithm for the linear canonical transform. The Journal of the Optical Society of America, 22, 928-937.

Jimenez, C., Torres, C., \& Mattos, L. (2011). Journal of physics: Conference series. International Journal of Computer Applications, 274.

Koc, A., Ozaktas, H. M., \& Hesselink, L. (2011). Fast and accurate algorithms for quadratic phase integrals in optics and signal processing. Three-Dimensional Imaging, Visualization, and Display: SPIE Proc Orlando, FL, 8043, 04-1-04-5.

Koç, A., Oktem, F. S., Ozaktas, H. M., \& Kutay, M. A. (2016). Linear canonical transforms: Theory and applications (pp. 293-327)). New York, NY: Springer New York.

Koç, A., Ozaktas, H. M., Candan, C., \& Kutay, M. A. (2008). Digital computation of linear canonical transforms. IEEE Transactions on Signal Processing, 56, 2383-2394.

Koc, A. Ozaktas, H. M., \& Hesselink, L. (2010a). Fast and accurate algorithm for the computation of complex linear canonical transforms. The Journal of the Optical Society of America, 27(9), 1896-1908.

Koç, A., Ozaktas, H. M., \& Hesselink, L. (2010b). Fast and accurate computation of two-dimensional non-separable quadratic-phase integrals. The Journal of the $\mathrm{Op}$ tical Society of America, 27(6), 1288-1302.

Kumar, R., Singh, K., \& Khanna, R. (2012). Satellite image compression using fractional fourier transform. International Journal of Computer Applications, 50(3), 0975-8887.

Leung, R., \& Taubman, D. (2005). Transform and embedded coding techniques for maximum efficiency and random accessibility in 3-d scalable compression. IEEE Transactions on Image Processing, 14, 1632-1646.

Li, B., \& Shi, Y. (2014). Image watermarking in the linear canonical transform domain. Mathematical Problems in Engineering.
Lustig, M., Donoho, D., \& Pauly, J. M. (2007). Sparse MRI: The application of compressed sensing for rapid MR imaging. Magnetic Resonance in Medicine, 58(6), $1182-1195$.

Ozaktas, H. M., Arıkan, O., Kutay, M. A., \& Bozdağı, G. (1996). Digital computation of the fractional fourier transform. IEEE Transactions on Signal Processing, 44, 2141-2150.

Ozaktas, H. M., Koç, A., Sari, I., \& Kutay, M. A. (2006). Efficient computation of quadratic-phase integrals in optics. Optics Letters, 31, 35-37.

Ozaktas, H. M., Zalevsky, Z., \& Kutay, M. A. (2001). The fractional fourier transform with applications in optics and signal processing. New York: Wiley.

Pei, S., \& Ding, J. (2002). Fractional cosine, sine, and hartley transforms. IEEE Transactions on Signal Processing, 50(7), 1661-1680.

Pennebaker, W. B., \& Mitchell, J. L. (1992). JPEG still image data compression standard. Kluwer.

Qiu, W., Li, B., \& Li, X. (2013). Speech recovery based on the linear canonical transform. Speech Communication, 55(1), 40-50.

Singh, N., \& Sinha, A. (2010). Chaos based multiple image encryption using multiple canonical transforms. Optics and Laser Technology, 42, 724-731.

Song, X., Peng, X., Xu, J., Shi, G., \& Wu, F. (2015). Cloud-based distributed image coding. IEEE Transactions on Circuits and Systems for Video Technology, 25(12), 1926-1940.

ISO/IEC 10918-1 || ITU-T Rec. T.81 (1992). Information Technology - Digital Compression and Coding of Continuous-tone Still Images.

ISO/IEC 15444-1 || ITU-T Rec. T.800 (2002). Information Technology - JPEG 2000 Image Coding System: Core Coding System.

Suzuki, T., \& Ikehara, M. (2010). Integer dct based on direct-lifting of dct-idct for lossless-to-lossy image coding. IEEE Transactions on Image Processing, 19(11), 2958-2965.

Wang, Z., Bovik, A. C., Sheikh, H. R., \& Simoncelli, E. P. (2004). Image quality assessment: From error visibility to structural similarity. IEEE Transactions on Image Processing, 13(4), 600-612.

Wolf, K. B. (1974). Canonical transformations I. Complex linear transforms. Journal of Mathematical Physics, 15, 1295-1301.

Zhu, S., \& Chen, J. (2012). Performance comparison of several transform domain image coding algorithms. In International conference on computer science and information processing (csip), 2012 (pp. 1030-1034). 